\title{
IGFBP7 functions as a potential lymphangiogenesis inducer in non-small cell lung carcinoma
}

\author{
WEIPENG ZHAO ${ }^{1-3}$, JUN WANG $^{4}$, BO ZHU $^{2}$, YUZHONG DUAN $^{2}$, FANGLIN CHEN $^{2}$, WEIQI NIAN ${ }^{2}$, \\ JIANGUO SUN ${ }^{2}$, BICHENG ZHANG ${ }^{5}$, ZHONGSHENG TONG ${ }^{1}$ and ZHENGTANG CHEN ${ }^{2}$
}

\author{
${ }^{1}$ Tianjin Medical University Cancer Institute and Hospital, National Clinical Research Center for Cancer, Key Laboratory of \\ Cancer Prevention and Therapy, Tianjin 300060; ${ }^{2}$ Institute of Cancer, Xinqiao Hospital, Third Military Medical University, \\ Chongqing 400037, P.R. China; ${ }^{3}$ Department of Immunology, Moffitt Cancer Center, Tampa, FL 33612, USA; \\ ${ }^{4}$ Department of Oncology, General Hospital, Jinan Command of the People's Liberation Army, Jinan, \\ Shandong 250031; ${ }^{5}$ Department of Oncology, Wuhan General Hospital of Guangzhou Command, \\ People's Liberation Army, Wuhan, Hubei 430070, P.R. China
}

Received October 3, 2015; Accepted November 14, 2015

DOI: 10.3892/or.2015.4516

\begin{abstract}
Lymphangiogenesis is not only involved in the processes of embryonic development, tissue repair and chronic inflammation, but also in tumor lymphatic metastasis. Metastatic tumor cells spreading through lymphatic vessels occur in non-small cell lung carcinoma (NSCLC), with regional lymph node metastasis often being the most important prognostic factor for carcinoma patients. Recent research has identified a range of lymphangiogenic growth factors that could conceivably play a great role in promoting tumor lymphangiogenesis and lymphatic metastasis. The most extensively accepted signaling pathways promoting lymphangiogenesis in tumors include the secreted lymphangiogenic proteins: vascular endothelial growth factor-C (VEGF-C) and VEGF-D, and their cognate receptor on lymphatic endothelium VEGF receptor-3 (VEGFR-3). Targeting VEGF pathway strategy sometimes failed to decrease tumor metastasis in vivo experiments and clinical trials. It is unclear whether the tumor cells induced the lymphangiogenesis process, while VEGF pathway could not completely illustrate the mechanism of tumor cell lymphatic metastasis. To explore the novel tumor lymphangiogenesis targets, we screened 181 candidate genes between high lymphatic vascular density (LVD) and low LVD in lung adenocarcinomas using Human Genome U133 Plus 2.0
\end{abstract}

Correspondence to: Dr Zhongsheng Tong, Tianjin Medical University Cancer Institute and Hospital, National Clinical Research Center for Cancer, Key Laboratory of Cancer Prevention and Therapy, Tianjin 300060, P.R. China

E-mail: tongzhongsheng@163.com

Dr Zhengtang Chen, Institute of Cancer, Xinqiao Hospital, Third Military Medical University, Chongqing 400037, P.R. China

E-mail:maoxun@gmail.com

Key words: Insulin-like growth factor binding protein 7, lymphangiogenesis, non-small cell lung carcinoma
Microarray. Insulin-like growth factor binding protein 7 (IGFBP7) was proven to be associated with metastatic clinicopathological features and high LVD. Furthermore, by assessing the capability of lymphatic endothelial cell forming lymphatic vessel-like structures in vitro, it appears to enhance lymphangiogenesis.

\section{Introduction}

Lung cancer, the leading cause of cancer morbidity, accounts for $13 \%$ (1.6 million) of total carcinoma cases and $18 \%$ (1.4 million) of the deaths in 2008 (1). Current treatments include surgery, radiotherapy, chemotherapy and immunotherapy. Multiple therapies improve the survival rate of non-small cell lung carcinoma (NSCLC) patients, but the median 5-year survival rate is still only $15 \%$ (2). The outcome of patients with NSCLC remains poor, and distant lymph node metastasis is one of the most important prognostic variables. Recent studies have suggested that lymphangiogenesis, the formation of new lymphatic vessels induced by tumors, was directly correlated with the extent of lymph node metastasis of solid tumors. The degree of lymphatic vessel density (LVD) could quantify tumor lymphangiogenesis. High LVD was correlative with poor outcome $(3,4)$.

Blood and lymphatic vessels are essential for the transport of fluids, gases, macromolecules and cells within the large and complex bodies of vertebrates (5). New blood and lymphatic vessels in tumors can grow by sprouting from pre-existing vessels or by recruitment of rare, circulating, bone marrow-derived endothelial progenitor cells $(6,7)$. The molecular basis of lymphangiogenesis is only partially understood due to an overlap between markers of endothelial cells (ECs) and lymphatic endothelial cells (LECs), the lack of specific lymphatic molecular markers, and the unavailability of good experimental models. The most studied mediators of lymphangiogenesis are the members of the vascular endothelial growth factor (VEGF) family, which are crucial players in the regulation of lymphangiogenesis as well as angiogenesis. 
High expression of VEGF-C and VEGF-D has been confirmed in clinicopathological studies, such as NSCLC, breast and colon cancer, malignant melanoma, head and neck and prostate cancer. Furthermore, LVD and lymphatic vessel invasion (LVI) are closely related to lymph node metastasis. With LEC isolation and establishment of lymphangiogenesis in an animal model, combined with some known molecular marker of LECs, such as VEGF receptor-3 (VEGFR-3), Prox-1, LYVE-1 and podoplanin, lymphangiogenesis study becomes a hot spot in tumor research (8). Our recent study showed that tumor cells overexpressing VEGF-C could induce lymphangiogenesis in surrounding tumor cells and promote invasion of lymphatic vessels, which is a key step in the metastasis of primary tumors to draining lymph nodes (9).

Insulin-like growth factor binding protein 7 (IGFBP7), a secreted $31-\mathrm{kDa}$ protein, is a related member of the IGFBP family (10). Unlike the other family members (IGFBP1-6), it exhibits a low affinity for IGF, but a high and specific affinity for insulin (11), and also confers a level of regulation to the IGF signaling system. It is also seen in endometrial glands expressing high levels of IGFBP7 during the mid-secretory phase of the menstrual cycle (12). Varied IGFBP7 expression patterns have been reported in different tumor types (13). Transient expression of the IGFBP7 protein in IGFBP7deficient cells blocks cell proliferation by causing senescence, apoptosis or delay of the cell cycle (14). A previous study provided evidence for pro-angiogenic function of IGFBP7 in human brain endothelial cells (HBECs) (15). In other studies, IGFBP7 was reported to act as a tumor-suppressor through the regulation of cell proliferation, cell adhesion, apoptosis, cellular senescence and angiogenesis (16). IGFBP7 accumulates in the capillary-like tubes of vascular endothelial cells in vitro (17). According to bioinformatics and biological function classification, IGFBP7 was included into the superfamily members of the extracellular matrix metalloproteases (3). In our study, to screen genes related to lymphangiogenesis in lung adenocarcinoma by Human Genome U133 Plus 2.0 Array, we established a differentially expressed cDNA library based on lymphatic vessel density. IGFBP7 was selected for further experiments in vivo and in vitro, based on the bioinformatics analysis and literatures review.

\section{Materials and methods}

Patients and tissues. The present study included 97 patients with NSCLC who underwent either lobectomy or pneumonectomy at Southwest Hospital, Xinqiao and Daping Hospitals, between February 2005 and February 2008. Among 97 samples, 34 lung adenocarcinoma samples were sliced into two parts, and one part was placed into the liquid nitrogen immediately, this subset was preserved for extracting mRNA and differential gene screening. The other part was formalinfixed, paraffin-embedded NSCLC tissues that were retrieved from the files of our pathology department. Pathological stage was re-evaluated and determined with the present TNM classification as revised in the WHO 2004 classification criteria. Tissue blocks containing a representative fraction of the tumor and the tumor-lung parenchyma interface were used. Operative tissues were also embedded with paraffin from the 97 patients with NSCLC. The study was approved by the Ethics Committee (Faculty of Medicine, Third Military Medical University).

Animals. Specific pathogen-free female C57BL/6 mice (4-6 weeks old) and female BALB/c mice (6 weeks old) were purchased from the Institute of Experimental Animal of Third Military Medical University (Chongqing, China). All animals had free access to standard laboratory mouse feed and water. The present study was conducted in accordance with the national and regional guidelines for the care and use of laboratory animals.

Cell lines. Murine Lewis Lung Cancer (LLC) and L929 (murine fibroblast) cells were purchased from American Type Culture Collection (ATCC; Manassas, VA, USA), and were maintained in Dulbecco's modified Eagle's medium containing 10\% fetal bovine serum (FBS) (both from Invitrogen, Carlsbad, CA, USA). Isolation and culture of LECs was performed as previously described (18). Briefly, female BALB/c mice were intraperitoneally injected with emulsified incomplete Freund's adjuvant (Sigma-Aldrich, St. Louis, MO, USA) to induce lymphangiomas. After 2 months of induction, tumors in the peritoneal cavity were removed and mechanically disrupted. LECs were isolated and resuspended in endothelial cell growth supplement (EBM-2; Cambrex BioScience, Wokingham, UK) with $20 \% \mathrm{FBS}$, and $50 \mathrm{ng} / \mathrm{ml}$ endothelial cell growth supplement (Cambrex BioScience) at $37^{\circ} \mathrm{C}$ in a humidified atmosphere of $5 \% \mathrm{CO}_{2}$. LECs were used in appropriate experiments or cultivated till the fourth passage/phase.

Co-cultivation and tube-like structure formation assay. LECs $\left(6 \times 10^{4}\right.$ cells/well) were put into a 24 -well plate that had been pre-coated with 100 Matrigel $(10 \mathrm{mg} / \mathrm{ml}$; Clontech, Palo Alto, CA, USA) and cultured for $24 \mathrm{~h}$. Transwell upper inserts were then placed into the co-cultivation system. The L929, LLC and Si-IGFBP7 LLC cells, respectively, were seeded into the Transwell upper inserts of chambers consisting of polycarbonate membranes (0.4-mm pore size; Millipore, Billerica, MA, USA). The cells in the upper inserts were LECs at $1 \times 10^{4}$ cells/well, and were co-cultured for $48 \mathrm{~h}$. Cells that were cultured in basal medium were used as a control. Formation of tube-like structures was monitored by microscopic observation at a magnification of $\mathrm{x} 100$ over 6 different fields of each well. They were then photographed to measure the length of tube-like structures as previously described (19).

Immunohistochemistry and LMVD. Serial 5- $\mu \mathrm{m}$ thick sections prepared from formalin-fixed, paraffin-embedded tissues from radical prostatectomy specimens were used for the study. Tissue blocks that contained the maximum amount of tumor and highest Gleason score were selected for each case, in order to ensure that the representative blocks contained cancer of the same Gleason score as the overall score of the case, but recognizing the limitation of sample variation. Slides from these representative blocks were analyzed. Slides were deparaffinized in xylene twice for $5 \mathrm{~min}$ and rehydrated through graded ethanol solutions to distilled water. Antigen retrieval was carried out by heating sections in $0.1 \mathrm{~mol} / \mathrm{l}$ citrate buffer, $\mathrm{pH}$ 6.0, in a pressure steamer for $20 \mathrm{~min}$. Endogenous peroxidase activity was inactivated by incubation in $3 \% \mathrm{H}_{2} \mathrm{O}_{2}$ 
for $15 \mathrm{~min}$. Non-specific binding sites were blocked using protein block (Dako Corp., Carpinteria, CA, USA) for $20 \mathrm{~min}$. The slides were then incubated sequentially with primary antibody (clone podoplanin, prediluted antibody; Abcam Inc., Cambridge, MA, USA), biotinylated secondary antibody, avidin-peroxidase complex and chromogenic substrate diaminobenzidine. Positive and negative controls were run in parallel with each batch and demonstrated that the procedure functioned properly (20).

At least 6 random fields per cross-section were visualized at a magnification of $\mathrm{x} 20$ and used for image analysis that was performed with the NIS-Elements Advanced Research 2.3 imaging software (image pro-plus), which identifies signals by threshold key intensity values. Furthermore, it permits imposing restrictions to the measurements by excluding false positive signals. Briefly, the number of positive cells expressing a particular antibody was calculated as a percent of the region of interest (ROI), as indicated in the individual figure legend. Co-localization of two antibodies was calculated by converting the area occupied by cells positive for the first antibody into a ROI. Then the percent of cells that were positive for the second antibody was calculated within the ROI (21).

Total RNA isolation and DNA microarray analysis. Total RNA was isolated from cultured cells using a single-step procedure with the TRIzol reagent (Invitrogen, Gaithersburg, MD, USA). The cells were lysed to extract the total RNA. The quality of total RNA was excellent, as deemed by measuring the 260/280 $\mathrm{nm}$ ratio (>2.0) and was then used for further cDNA synthesis and a chip hybridization procedure according to the manufacturer's instructions (HU133 Plus 2; Affymetrix, Inc., Santa Clara, CA, USA). The microarray data were analyzed at the NetAffx Analysis Center within the Affymetrix website, http://www.affymetrix.com/analysis/ index.affx. cDNA was synthesized from $5 \mu \mathrm{g}$ of total RNA with 200 U of MMLV-RT (Promega, Madison, WI, USA) and $500 \mathrm{ng}$ of a 16 -mer oligo(dT). All the primer sequences for the PCR reactions are listed in Table I and the cycling conditions for all of the PCRs were 30 cycles of a denaturation period $\left(94^{\circ} \mathrm{C} / 30 \mathrm{sec}\right)$ that was followed by an annealing period $\left(55^{\circ} \mathrm{C} / 30 \mathrm{sec}\right)$, and an extension period $\left(72^{\circ} \mathrm{C} / 40 \mathrm{sec}\right)$, then a final extension period $\left(72^{\circ} \mathrm{C} / 10 \mathrm{~min}\right)$, except for $\beta$-actin which contained an annealing period of $60^{\circ} \mathrm{C} / 30 \mathrm{sec}$ in a thermal cycler (Bio-Rad MJ Research PCR; Bio-Rad, Waltham, MA, USA). PCR products were separated on a $1.5 \%$ agarose gel in a $0.5 \mathrm{X}$ TBE buffer and were stained with ethidium bromide and visualized under UV light.

Microarray hybridization. Purified total RNA (5 Ag) was labeled and hybridized onto the Affymetrix U133 Plus 2.0 GeneChip oligonucleotide arrays (Affymetrix, Inc.) according to the manufacturer's instructions. Briefly, hybridization signals were scaled in Affymetrix GCOS software (version 1.1.1) using a scaling factor determined by adjusting the global trimmed mean signal intensity value to 500 for each array and importing them into GeneSpring version 6.2 (Silicon Genetics, Redwood City, CA, USA). Signal intensities were then centered to the 50th percentile of each chip, and for each individual gene, to the median intensity of each specific subset first to minimize the possible technical bias and then to the
Table I. Association of LVD with clinicopathological features in lung adenocarcinoma.

\begin{tabular}{|c|c|c|c|}
\hline $\begin{array}{l}\text { Clinicopathological } \\
\text { features }\end{array}$ & Cases & ptLVD & itLVD \\
\hline \multicolumn{4}{|l|}{ Age (years) } \\
\hline$\geq 54.5$ & 17 & $23.1 \pm 7.9$ & $10.3 \pm 5.0$ \\
\hline$<54.5$ & 17 & $21.7 \pm 8.1$ & $14.2 \pm 4.9$ \\
\hline \multicolumn{4}{|l|}{ Gender } \\
\hline Male & 21 & $21.5 \pm 7.5$ & $10.2 \pm 4.7$ \\
\hline Female & 13 & $21.3 \pm 7.7$ & $9.2 \pm 4.5$ \\
\hline \multicolumn{4}{|l|}{ Tumor differentiation } \\
\hline Well-moderate & 25 & $22.3 \pm 8.1$ & $10.2 \pm 6.4$ \\
\hline Poor & 9 & $21.7 \pm 8.1$ & $10.7 \pm 5.4$ \\
\hline \multicolumn{4}{|l|}{ Pathologic N stage } \\
\hline $\mathrm{N} 1-2$ & 16 & $24.3 \pm 8.7^{b}$ & $11.3 \pm 5.5$ \\
\hline N0 & 18 & $18.4 \pm 6.3$ & $11.7 \pm 4.3$ \\
\hline \multicolumn{4}{|l|}{$\mathrm{ptLVD}^{\mathrm{a}}$} \\
\hline High $(\geq 18.7)$ & 17 & - & $12.6 \pm 6.1$ \\
\hline Low $(<18.7)$ & 17 & - & $12.1 \pm 4.3$ \\
\hline \multicolumn{4}{|l|}{$\mathrm{itLVD}^{\mathrm{a}}$} \\
\hline $\operatorname{High}(\geq 10.5)$ & 17 & $23.9 \pm 7.1$ & - \\
\hline Low $(<10.5)$ & 17 & $21.3 \pm 6.5$ & - \\
\hline \multicolumn{4}{|l|}{ Pathological stage } \\
\hline I-II & 19 & $18.4 \pm 5.6$ & $10.2 \pm 4.4$ \\
\hline III-IV & 15 & $24.1 \pm 8.2^{\mathrm{b}}$ & $10.3 \pm 3.4$ \\
\hline
\end{tabular}

${ }^{\mathrm{a} C u t}$-off value is the median value. ${ }^{\mathrm{b}} \mathrm{Correlation}$ is statistically significant, $\mathrm{P}<0.05$. LVD, lymphatic vascular density; ptLVD, peritumoral lymphatic vessel density; itLVD, intratumoral lymphatic vessel density.

whole sample set. The intensities of any replicate hybridizations were averaged subsequent to further analysis.

Real-time PCR confirmation of a selection of differentially expressed genes. Relative gene copy numbers and gene expression was determined by quantitative real-time PCR using a PRISM 7500 Sequence Detection system (Applied Biosystems) and a QuantiTect SYBR-Green PCR kit (Qiagen, Inc. Valencia, CA, USA). The standard curve method was used to calculate target gene copy number in the tumor cDNA sample normalized to a repetitive element line-1 and normal reference cDNA. The comparative threshold cycle method was used to calculate gene expression normalized to $\beta$-actin as a gene reference and normal human lung RNA was used as an RNA reference. Primers were designed using Primer3 (http://frodo.wi.mit. edu/cgi-bin/primer3/primer3_www.cgi) and were synthesized by Sangon Biotech Co., Ltd. (Shanghai, China). Primers (Invitrogen, Shanghai, China) used for RT-PCR were: IGFBP7 5'-CTGGGTGCTGGTATCTCCTC-3' (sense), and 5'-TATAG CTCGGCACCTTCACC-3' (antisense); VEGF-C 5'-TGTAAA ACGACGGCCAGT-3' (sense), and 5'-CAGGAAACAGCTAT GACC-3' (antisense); GAPDH 5'-GCACCGTCAAGGCTG AGAAC-3' (sense), and 5'-TGGTGGTGAAGACGCCAGT-3' (antisense). 


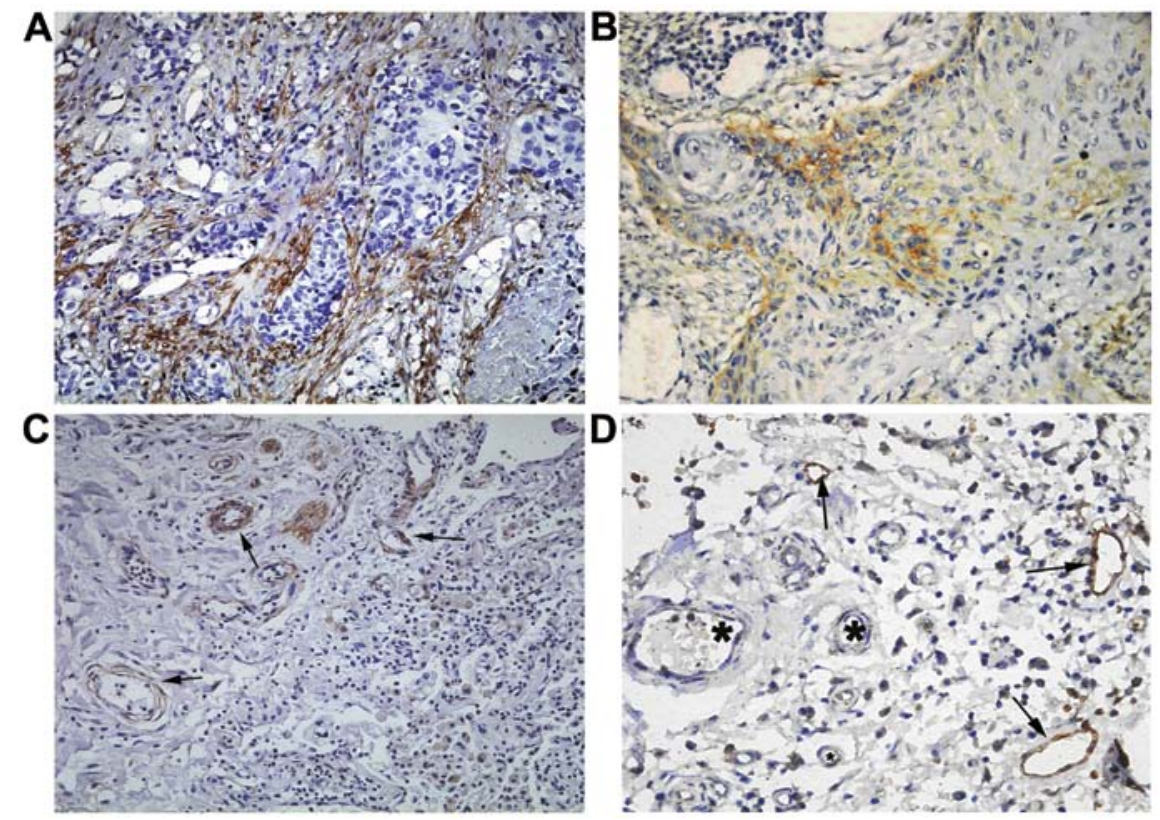

Figure 1. Morphology of lymphatic vessels in lung adenocarcinoma tissue. (A) The periphery tumor lymph vessels of the lung adenocarcinoma, magnification, x200. (B) The intratumoral lymph vessels of the lung adenocarcinoma, magnification, x200. (C) The peritumoral lymphatics in low LVD group, magnification, x200. (D) Some blood vessels (star) were not stained by the podoplanin antibody, but were stained for lymph vessels $(\rightarrow)$, magnification, x400.

Protein pathway analysis. Gene Ontology (GO) annotation of the identified proteins was obtained using DAVID (version 6.7); (see http://david.abcc.ncifcrf.gov/summary.jsp.) Differentially expressed proteins were analyzed using Ingenuity Pathway Analysis (IPA; Ingenuity Systems; see www.ingenuity.com). Cell Death, Cellular, Development, Hematological Systems Development and Function, Endocrine System, Disorders, Metabolic Disease, Cell-To-Cell Signaling interactions were generated based on information contained in the Ingenuity Pathways Knowledge Base.

Western blotting. Biological replicates were used for western blot analysis of astroglial differentiation. In brief, lysates from whole cell extracts or membrane pellets containing $20 \mu \mathrm{g}$ of proteins were subjected to gel electrophoresis. Proteins were then transferred to PVDF membranes (Millipore). The blots were blocked in 4\% BSA in TBST solution for $30 \mathrm{~min}$ at room temperature and were then incubated at $4^{\circ} \mathrm{C}$ overnight with the primary antibody. Anti- $\beta$-actin $(1: 4,000)$ and IGFBP7 $(1: 1,000)$ were purchased from Abcam, Inc. After incubation with secondary antibodies (1:2,000, Millipore) at room temperature for $1 \mathrm{~h}$, the blot was visualized by ChemiDoc XRS imaging system (Bio-Rad, Hercules, CA, USA).

Small interfering RNA transfection. Transfection with IGFBP7 siRNA (10 pmole) and irrelevant scrambled control siRNA was performed using Lipofectamine 2000 (Invitrogen) according to the manufacturer's protocol. LLCs at $50 \%$ confluency were treated with siRNA for $24 \mathrm{~h}$ in medium lacking $20 \%$ serum and antibiotics. Inhibition of IGFBP7 protein expression was observed within $24 \mathrm{~h}$ and this knockdown was maintained for at least $72 \mathrm{~h}$ after removal of the medium containing the siRNA.

Statistical analysis. Three statistical methods were used to identify the optimal prognostic gene signature: Cox proportional-hazard regression modeling, bootstrapping, and a 2 -fold cross-validation. Hierarchical clustering was done to identify major clusters of gene activation and investigate their associations with patient covariates. The method for developing a risk index is similar to that previously described. Pathway analysis was carried out by first mapping genes to the biological process categories of GO and then calculating the significance of overrepresented categories in the selected gene list. Correlations between podoplanin and the vessel numbers as continuous variables were used to determine positive vessel counts with the Spearman rank correlation test. Categorical data was compared by the $\chi^{2}$ or Fishers' exact probability test. Distribution was normal or within the Mann Whitney U test if the sample distribution was asymmetrical. The relationship between lymph vessel variables and lymph node status was analyzed by one-way ANOVA, followed by the Neuman Keuls test.

\section{Results}

Morphology of lymphatic vessels in lung adenocarcinoma tissue. Podoplanin expression mostly presented in the thinwalled structures. Podoplanin was positive in endothelial cell plasma, in thin-walled lymph vessel, as indicated by the brown-yellow color. Podoplanin positive lymph vessels were both located at the adenocarcinoma interstitium (Fig. 1A) and at the tumor boundary (Fig. 1B). In some cases, low density lymphatic vessels (LVD- $-_{\text {low }}$ ) appear around tumor cells (Fig. 1C). Podoplanin positive stained cells only appear in thin-walled structures like the lymphatic vessel, but does not stain blood vessels, which are indicated by the presence of blood cells (Fig. 1D).

Association of LVD with clinicopathological features. Table I depicts pathologic N1-2 as significantly different with No 
Table II.

\begin{tabular}{|c|c|c|c|}
\hline Gene symbol & GenBank & Fold-change & Description \\
\hline \multicolumn{4}{|c|}{ Top upregulated genes } \\
\hline LOC387601 & AK091990 & 38.6 & Putative UST1-like organic anion transporter \\
\hline ANXA8 & NM_001630 & 14.89 & Annexin A8 \\
\hline SLC1A2 & AV722518 & 11.7 & Solute carrier family 1 (glial high affinity glutamate transporter), member 2 \\
\hline PAPSS2 & AI821404 & 9.784 & 3'-Phosphoadenosine 5'-phosphosulfate synthase 2 \\
\hline IGFBP7 & AU144916 & 8.95 & Insulin-like growth factor binding protein 7 \\
\hline DOCK1 & AK000789 & 5.441 & Dedicator of cytokinesis 1 \\
\hline ATP5S & BE968806 & 5.223 & ATP synthase, $\mathrm{H}^{+}$transporting, mitochondrial F0 complex, subunits (factor B) \\
\hline RPL23 & AK025200 & 4.651 & Ribosomal protein L23 \\
\hline ATP1B3 & AI928218 & 4.508 & ATPase, $\mathrm{Na}^{+} / \mathrm{K}^{+}$transporting, $\beta 3$ polypeptide \\
\hline LOC 152573 & AI735586 & 3.805 & Hypothetical protein BC012029 \\
\hline CD1E & AA309511 & 3.568 & CD1E antigen, e polypeptide \\
\hline CCNA1 & NM_003914 & 3.528 & Cyclin A1 \\
\hline STXBP1 & NM_003165 & 3.506 & Syntaxin binding protein 1 \\
\hline C9orf3 & AI147867 & 3.44 & Chromosome 9 open reading frame 3 \\
\hline ZDHHC21 & BE467787 & 3.289 & Zinc finger, DHHC-type containing 21 \\
\hline \multicolumn{4}{|c|}{ Top downregulated genes } \\
\hline GMDS & AK000788 & 0.227 & GDP-mannose 4,6-dehydratase \\
\hline CDH1 & L08599 & 0.224 & Cadherin 1, type 1, E-cadherin (epithelial) \\
\hline CCL28 & AF266504 & 0.199 & Chemokine (C-C motif) ligand 28 \\
\hline TNFRSF21 & NM_016629 & 0.196 & Tumor necrosis factor receptor superfamily, member 21 \\
\hline BMP5 & AK021486 & 0.193 & Bone morphogenetic protein 5 \\
\hline AP1S3 & AI474433 & 0.174 & Adaptor-related protein complex 1 , sigma 3 subunit \\
\hline HSPC105 & AI914083 & 0.154 & NAD(P) dependent steroid dehydrogenase-like \\
\hline PRLR & S78505 & 0.154 & Prolactin receptor \\
\hline FLJ14503 & AW237462 & 0.126 & Hypothetical protein FLJ14503 \\
\hline OPRK1 & AU153412 & 0.0987 & Opioid receptor, $\kappa 1$ \\
\hline C18orf2 & AF295726 & 0.0783 & Chromosome 18 open reading frame 2 \\
\hline KIAA1324 & AI672868 & 0.0556 & KIAA1324 \\
\hline CRISP2 & M25532 & 0.0233 & Cysteine-rich secretory protein 2 \\
\hline MAGEA6 & U10691 & 0.0118 & Melanoma antigen family A, 6 \\
\hline CRISP3 & NM_006061 & 0.00807 & Cysteine-rich secretory protein 3 \\
\hline MAGEA3 & ВC000340 & 0.0047 & Melanoma antigen family A, 3 \\
\hline
\end{tabular}

patients in assessing peritumoral lymphatic vessel density, but not intratumoral lymphatic vessel density $(\mathrm{P}<0.05)$. PtLVD was $24.1 \pm 8.2 /$ field in clinic pathlogical stage III-IV, which was significantly different with stage I-II $(\mathrm{P}<0.05)$, the mean of ptLVD is $18.4 \pm 5.6 /$ field. The median ptLVD was $18.7 /$ field, which is separated by $\mathrm{LVD}_{-}{ }_{\text {high }}$ and $\mathrm{LVD}_{-}{ }_{\text {low }}$ group. The clinicopathological features show that for ptLVD there was no significant difference with age, gender or tumor differentiation. No significant association was found with ptLVD or any of the clinicopathological criteria.

Genes differentially expressed between high ptLVD and low ptLVD lung carcinoma tissue. With analyzing association of LVD with clinicopathological feature, the three high ptLVD and low ptLVD lung carcinoma tissues were chosen for microarray screening. Using the criteria described in Materials and methods for microarray data analysis, we found 181 genes which showed a 2-fold difference. Among these, 97 genes are upregulated in high lymphatic vessels and 84 were downregulated. Upregulated and downregulated genes are reported in Table II. For each gene symbol, GenBank ID, fold-change and description are reported. Based on bioinformatics analysis and their respective functions reported in the literature, we selected 10 differentially expressed genes of interest and determined the expression levels by quantitative real-time RT-PCR. RT-PCR among these 10 genes demonstrated IGFBP7 to be the most differentially expressed. Thus, we chose to focus on IGFBP7 in the present study, whereas other candidates are being investigated in additional studies (Fig. 2).

Bioinformatics analysis of the differentially expressed membrane proteins. The DAVID Bioinformatics Resource 6.7 was used for annotation of the cellular component. IPA was used to assign identified proteins into different functional 

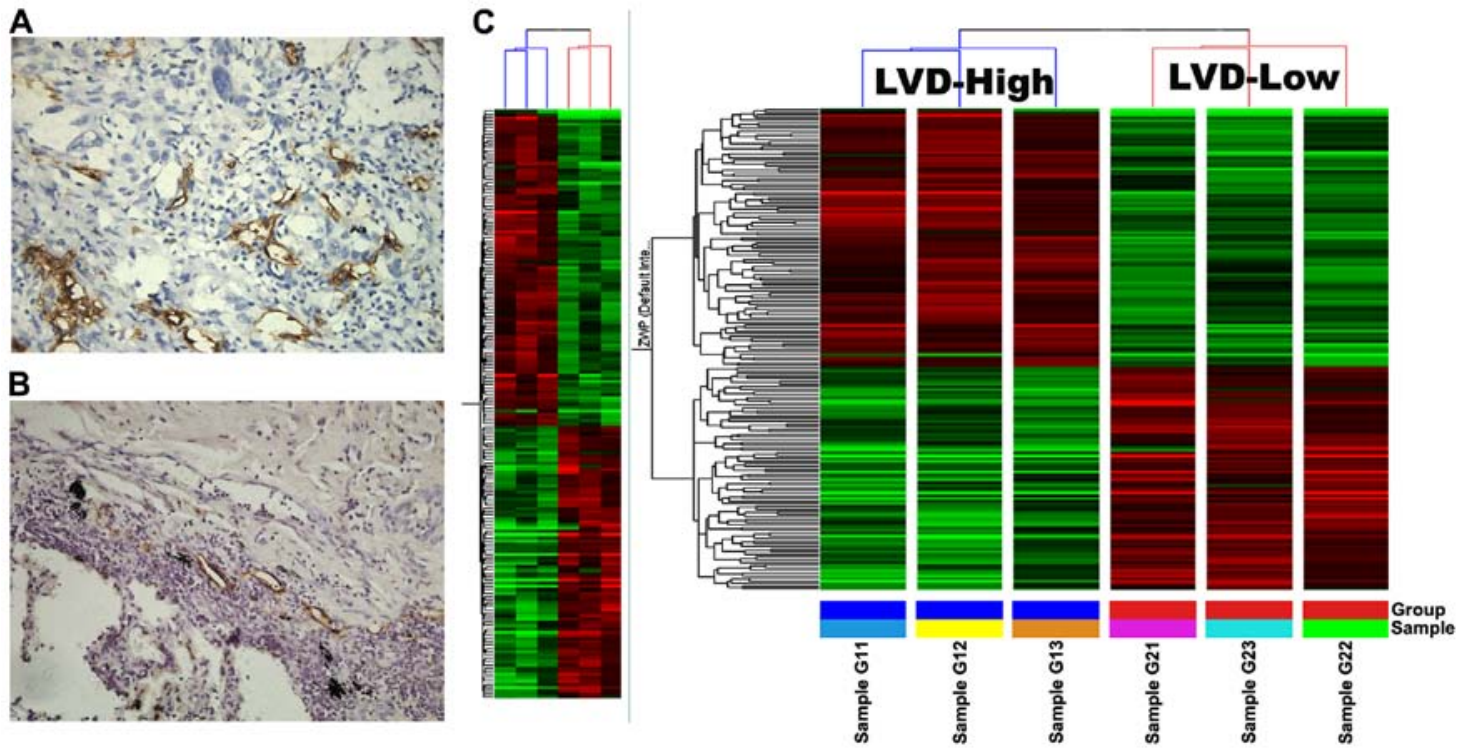

Figure 2. Identification of a gene expression signature to classify different lymhangiogenesis. (A and B) The periphery tumor lymph vessels of the lung adenocarcinoma, magnification, $x 200$, between $\mathrm{LVD}_{- \text {high }}$ and $\mathrm{LVD}_{-}-_{\text {low }}$ were stained by podoplanin. (C) Microarray and hierarchical cluster analyses of the top 181 differentially expressed genes between LVD- ${ }_{\text {high }}$ and LVD- ${ }_{- \text {low }}$. A large number of genes show distinct expression patterns between LVD- $_{\text {high }}$ and LVD-low. The colors indicate the relative gene expression level within the genes across all samples. Red, high expression; green, low expression. See Materials and methods for details.
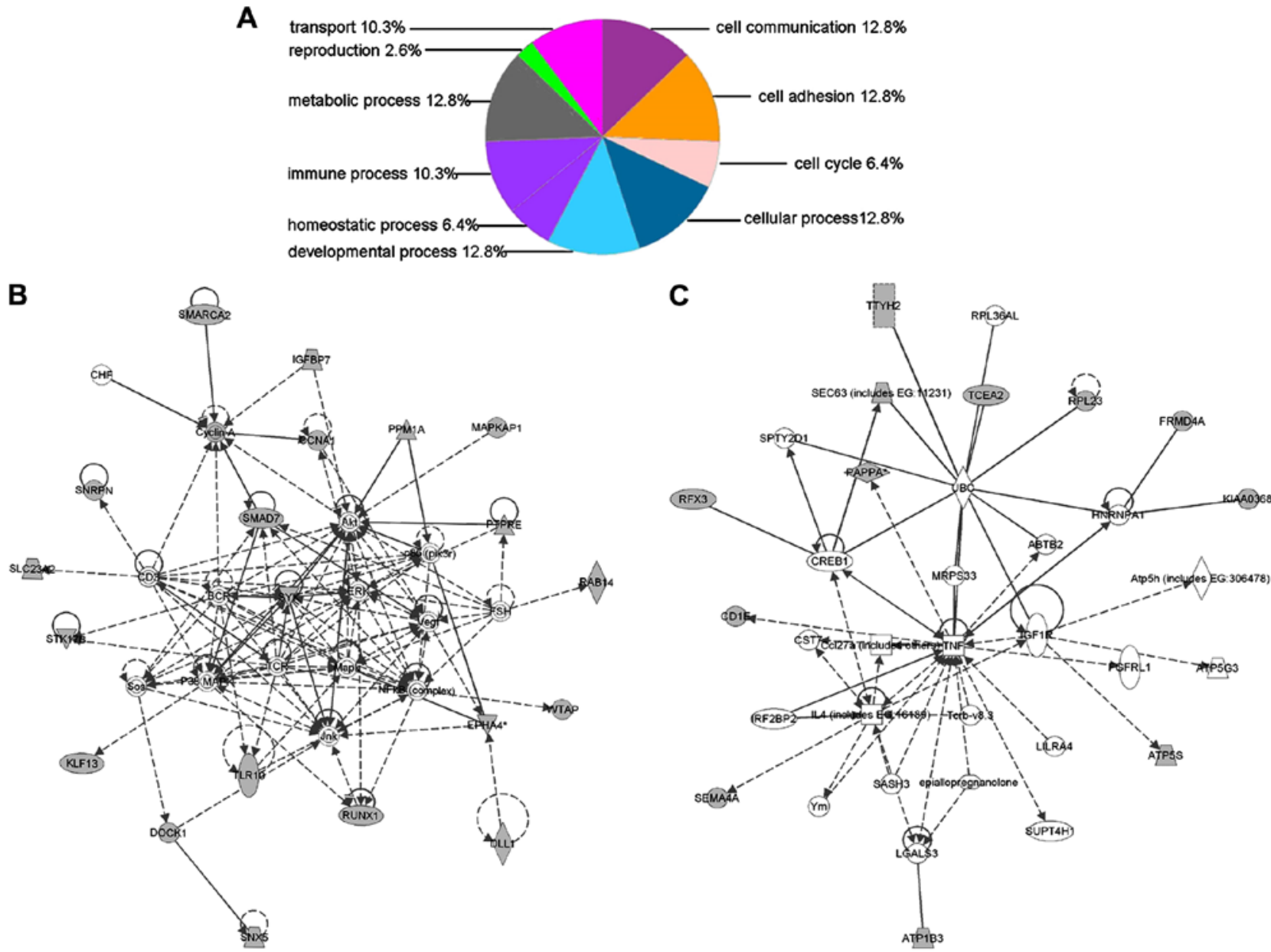

Figure 3. Molecular pathway analysis of regulated membrane protein changes in high LVD group. (A) The distribution of annotation for the cellular components for differentially expressed proteins; the cellular location was determined by GO annotations from the DAVID database. Proteins, and their expression levels of change, were imported into the Ingenuity Pathways Analysis, and interacting pathways were constructed. (B) Network 1 grouped by IPA shows it was primarily involved in Cell Death, Cellular, Development, Hematological System Development and Function. (C) Network 2 grouped by IPA shows it was involved in the Endocrine System, Disorders, Metabolic Disease Cell-To-Cell Signaling and interaction. 


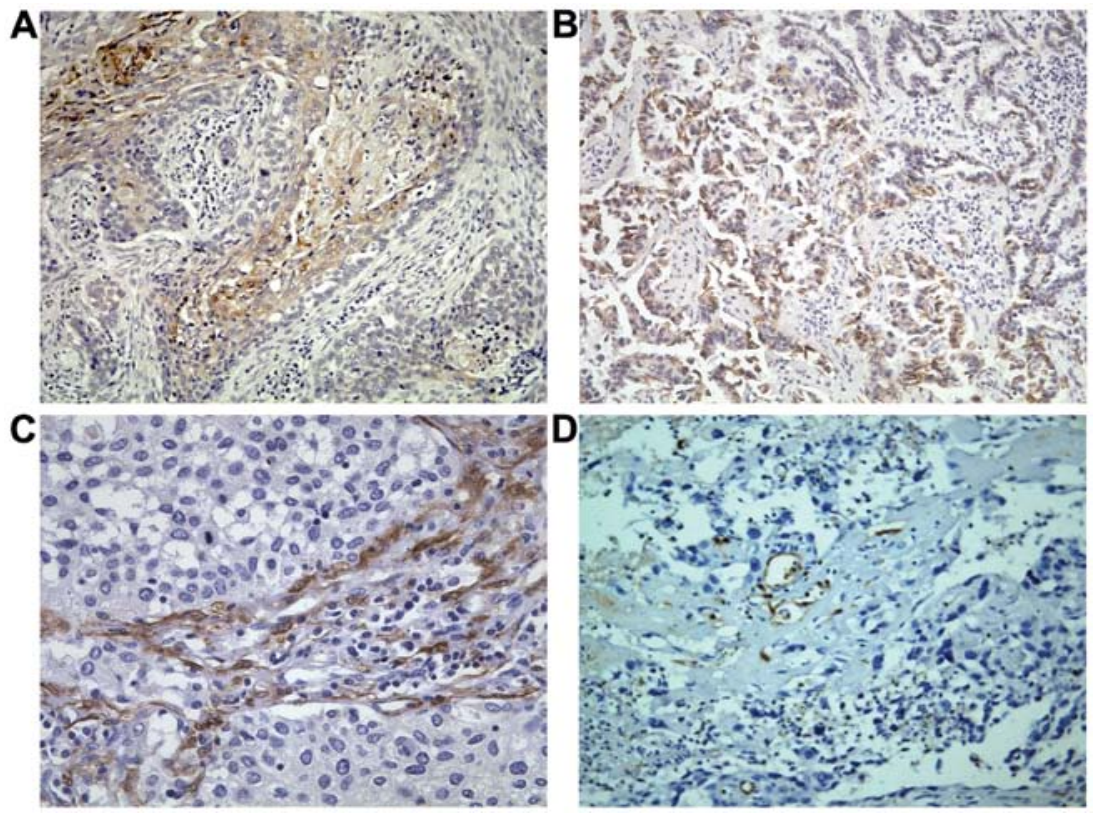

Figure 4. Expression of IGFBP7 in adenocarcinoma and squamous cell carcinoma. (SP, magnification, x200). (A) IGFBP7 antigen expression in lung squamous carcinoma. (B) IGFBP7 antigen expression in lung adenocarcinoma. (C) Lymphatic vessels stained by podoplanin in lung squamous carcinoma. (D) Lymphatic vessels stained by podoplanin in lung adenocarcinoma; magnification, $\mathrm{x} 200$.

groups based on the Ingenuity Pathways Analysis literature database. All the differentially expressed proteins were uploaded to the IPA server. For molecular and cellular functions, the data indicated that many proteins were involved in top 10 biological processes (Fig. 3A). Pathway analysis was also used to analyze different cellular functional networks to determine which were altered in the lymphangiogenesis procedure. In the present study, several networks were grouped by IPA, i.e. there are 20 proteins involved in cell death, cellular, development, hematological system development and function were grouped as the top network (network 1), which had the highest score (47) (Fig. 3B). Additionally, network 2 grouped by IPA has 12 related genes involved in endocrine system, disorders, metabolic disease, cell-to-cell signaling and interaction, and had a score of 25 (Fig. 3C).

Association of IGFBP7 with clinicopathological variables. In the NSCLC tissue, moderate IGFBPP7 immunoreactivity was present in the cytoplasm of lung cancer cells; a weak immunoreactivity was seen in some ductal cells within the small ductules. IGFBP7 positive substances that were in the shape of brownish-yellow fine particles were mainly located in the cytoplasm of the cancer cells. There was expression of various degrees on the cell membrane, presenting pale-yellow to brownish-yellow (Fig. 4).

The associations of high expression levels of IGFBP7 with clinicopathological parameters are shown in Table III. The positive expression of IGFBP7 is $55.67 \%$ (54/97). We observed a significant association between IGFBP7 expression in NSCLC associated with increased lymph node metastasis $(\mathrm{P}=0.002)$. Furthermore, ptLVD of the positive IGFBP7 is $23.1 \pm 8.5 /$ field, statistically significantly higher than the negative group $(16.9 \pm 6.0)$. There were no statistically significant differences with regard to patient age, gender or histological types (Table III).
Table III. Correlation of clinicopathologic features and expression of IGFBP7 in patients with non-small cell lung carcinoma.

\begin{tabular}{lccc}
\hline \multirow{2}{*}{$\begin{array}{l}\text { Clinicopathological } \\
\text { factors }\end{array}$} & \multicolumn{3}{c}{ Expression of IGFBP7 } \\
\cline { 2 - 4 } Age (years) & Negative & Positive & P-value \\
$\leq 65$ & 25 & 34 & 0.679 \\
$>65$ & 18 & 20 & \\
Gender & & & \\
Male & 30 & 32 & 0.298 \\
Female & 13 & 22 & \\
Histologic type & & & \\
Squamous carcinoma & 27 & 30 & 0.536 \\
Adenocarcinoma & 16 & 24 & \\
Differentiation & & & \\
Well & 35 & 37 & 0.169 \\
Poorly & 8 & 17 & \\
Pathological stages & & & \\
I+II & 29 & 39 & 0.659 \\
III+IV & 14 & 15 & \\
Pathologic N factor & & & \\
N0 & 9 & 26 & $0.002^{\mathrm{a}}$ \\
N1+N2+N3 & 34 & 28 & $0.001^{\mathrm{b}}$ \\
ptLVD & $16.9 \pm 6.0$ & $23.1 \pm 8.5$ & \\
\hline
\end{tabular}

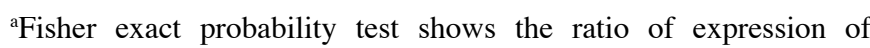
IGFBP7 in N0 is lower than in $\mathrm{N} 1+\mathrm{N} 2+\mathrm{N} 3(\mathrm{P}<0.05) .{ }^{\mathrm{b}}$ Analysis by the Spearman test shows positive expression of IGFBP7 ptLVD is higher than the negative $(\mathrm{P}<0.05)$. ptLVD, peritumoral lymphatic vessel density. 
A

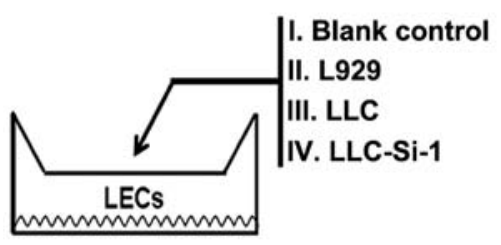

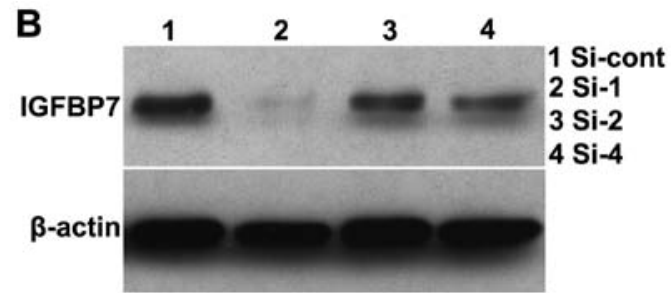

IGFBP7

D

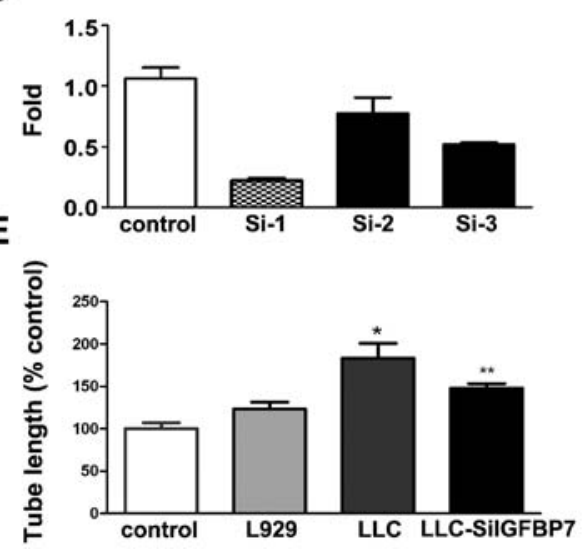

Figure 5. The effect of IGFBP7 on tube-like structure formation of LECs. (A) I, no cells in upper chamber; II, L929 cells in upper chamber; III, Lewis lung cancer cells in upper chamber; IV, LLC with IGFBP7 knocked down with LECs co-culture. (B) LLCs with IGFBP7-Si-control, Si-1, Si-2 and Si-3 were qualified by IGFBP7 western blotting. (D) RT-PCR confirmed IGFBP7-Si-control, Si-1, Si-2 and Si-3 expression. (C and E) Among the different cells co-cultured, the number of the intersecting branches of assembled endothelial cell networks was observed at a magnification of $\mathrm{x} 200$. Branch point numbers were calculated as the mean $\pm \mathrm{SD} .{ }^{*} \mathrm{P}<0.05$, LLC vs. L929; ${ }^{* *} \mathrm{P}<0.05$, LLC vs. LLC-SiIGFBP7.

The effect of IGFBP7 on tube-like structure formation of LECs. In the co-culture system, different cells were placed in the upper chambers, while LECs were in the lower chambers. After $48 \mathrm{~h}$ of LLC-LECs co-culture, LECs start establishing a complex tube system. After $72 \mathrm{~h}$ of culture in conditioned medium, the number of LECs permeating septum in the LLC group was the highest among the four groups. LLCs more strongly enhanced the formation of extensive capillary-like structures in vitro than medium alone or the L929 cells group $(\mathrm{P}<0.05)$. Furthermore, compared to the LLC group, the LLC-Si-IGFBP7 group decreased LEC formation of capillarylike structures in vitro $(\mathrm{P}<0.05)$ (Fig. 5).

\section{Discussion}

In recent years, lymphangiogenesis in pulmonary cancer constitutes a topic of intense study. The importance of lymph node metastases in predicting the course of neoplastic disease focuses our attention on understanding how tumors interact with the lymphatic vasculature (22). Our team used metaanalysis of the literature on lymphatic microvessel density in NSCLC. The lymphatic microvessel count or LVD, which reflects the level of lymphangiogenesis, is a strong indicator of poor prognosis for patient survival in surgically treated NSCLC (23).

The most extensively studied molecular system that signals for tumor lymphangiogenesis, and is associated with lymphatic spread from primary cancers, is the VEGF-C/VEGF-D/ VEGFR-3 signaling axis (24). Although lymph node metastasis occurs frequently in lung adenocarcinoma, size of the primary tumor and occurrence of distant metastasis is not parallel in many clinic cases. However, the VEGF-C/VEGF-D/VEGFR-3 signaling axis can not completely explain the complicated lymphangiogenesis in tumor cell metastasis. To investigate the related genes involved in lymphangiogenesis in NSCLC, we first assessed both intratumoral and peritumoral lymphatic vessel density in 34 lung adenocarcinomas. The threshold number was 18.7 between the high ptLVD and the low ptLVD group. Human Genome Microarray was employed for comparison of expression profiles of $\mathrm{LVD}_{- \text {high }}$ lung adenocarcinoma and LVD- -ow lung adenocarcinoma. We found 181 genes which were 2-fold differentially changed. In other studies, IGFBP7 was screened in the microarray $(25,26)$, which may be related with tumor metastasis. By identification and quantification of membrane proteins, network 2 (grouped by IPA) shows 12-related genes involved in the endocrine system, disorders, metabolic disease cell-to-cell signaling and interaction.

IGFBP7 expression in peripheral endothelial cells can be modulated by cell confluence, hypoxia and cytokines including VEGF, bFGF and TGF-b1. Secreted IGFBP7 is reported to interact with the ECM components, and could thus participate in the TGF-b1-induced extracellular matrix turnover and angiogenesis (27). Assessing the immunohistochemistry result of IGFBP7 staining, together with a previous study (28), our data show that IGFBP7 expression in NSCLC is associated with increased lymph node metastasis In other studies, esophageal adenocarcinoma exhibits the IGFBP7 expression pattern expected in an aggressive form of cancer (29). Overexpression of IGFBP7 was shown at the mRNA level in two independent Sporadic Pilocytic Astrocytomas tumor series (30). 
Furthermore, IGFBP7 plays a positive, contributing role in the interaction between leukemia cells and the microenvironment, which may promote the leukemic cell adhesion, invasion and migration (31). Another assay with IGFBP7 knockdown and acute lymphoblastic leukemia (ALL), showed that IGFBP7 acts as a positive regulator of ALL and stromal cell growth, and significantly enhances in vitro resistance of ALL to sparaginase (32). Hypomethylation of IGFBP7 is likely to characterize an immature and a more malignant subtype of the disease (33). In glioblastoma, developed anti-IGFBP7-iron oxide single domain antibody-targeted MRI contrast agent selectively binds to abnormal vessels (34), IGFBP7 antibody are novel glioblastoma vessel-targeting moieties suitable for molecular imaging (35), the retinal phenotype appears to be mediated by a role in the vascular endothelium, where IGFBP7 is highly expressed (36). In lung cancer patients, high serum levels of IGFBP7 is correlated with positive nodal status $(\mathrm{P}=0.008)$, thus is not beneficial for recurrence-free survival (37), in vitro IGFBP7 is only reported during cancer progression and metastasis formation (38).

As endothelial IGFBP7 induction by the tumor microenvironment is predominantly mediated by the ALK5/Smad-2 pathway, IGFBP7 accumulates in the basal lamina of GBM vessels in vivo (15). We propose that IGFBP7 may be involved in lymphangiogenesis in the context of NSCLC. As there is currently no clear understanding of whether lymphangiogenesis contributes to tumor progression or tumor growth stabilization, the overall effect of IGFBP7 in the tumor milieu cannot be easily generalized. As a secreted protein, IGFBP7 may also modulate the function of the immune system generating an antitumor immune response; this hypothesis remains to be experimentally tested. The proposed angiogenic role of IGFBP7 may appear contradictory to literature evidence of the tumor-suppressor activity of IGFBP7 $(14,39)$. The main explanation is that IGFBP7 could trigger differential signaling pathways in tumor and endothelial cells.

In summary, we identified IGFBP7 as enhancer of lymphangiogenesis in NSCLC, therefore, we believe it is a good target for inhibition. Our preliminary findings document that IGFBP7 efficiently enhances tube-like structure formation of LEC. Studies are ongoing to develop targeted and effective delivery systems for administering IGFBP7 in patients.

\section{Acknowledgements}

The present study was supported by the National Natural Science Foundation of China (grant no. 81472183). We thank Crystina Bronk for revising the manuscript.

\section{References}

1. Jemal A, Bray F, Center MM, Ferlay J, Ward E and Forman D: Global cancer statistics. CA Cancer J Clin 61: 69-90, 2011.

2. Molina JR, Adjei AA and Jett JR: Advances in chemotherapy of non-small cell lung cancer. Chest 130: 1211-1219, 2006

3. Kadota K, Huang CL, Liu D, Nakashima N, Yokomise H, Ueno $M$ and Haba R: The clinical significance of the tumor cell D2-40 immunoreactivity in non-small cell lung cancer. Lung Cancer 70: 88-93, 2010

4. Gao Y, Liu Z, Gao F and Meng XY: High density of peritumoral lymphatic vessels is a potential prognostic marker of endometria carcinoma: A clinical immunohistochemical method study. BMC Cancer 10: 131, 2010.
5. Chen W, Li Y, Liao Z, Lin G, Cai G, Lin K, Zhan Q and Chen C: Active lymphangiogenesis is a major risk factor for anastomotic leakage following sphincter-sparing resection of rectal cancer. J Surg Oncol 104: 493-498, 2011.

6. Chen D, Zheng J, Li H, Wang Q and Jiao X: Computer-assisted morphometric analysis of lymphatic vessel changes in hamster tongue carcinogenesis. J Oral Pathol Med 39: 518-524, 2010.

7. Sasahira T, Kirita T, Kurihara M, Yamamoto K, Bhawal UK, Bosserhoff AK and Kuniyasu H: MIA-dependent angiogenesis and lymphangiogenesis are closely associated with progression, nodal metastasis and poor prognosis in tongue squamous cell carcinoma. Eur J Cancer 46: 2285-2294, 2010.

8. Vittet D and Feige JJ: Lymphangiogenesis and tumor progression]. Bull Cancer 94: 881-886, 2007 (In French).

9. Sun JG, Wang Y, Chen ZT, Zhuo WL, Zhu B, Liao RX and Zhang SX: Detection of lymphangiogenesis in non-small cell lung cancer and its prognostic value. J Exp Clin Cancer Res 28: $21,2009$.

10. Heinzelbecker J, Kempf KM, Kurz K, Steidler A, Weiss C, Jackson DG, Bolenz C, Haecker A and Trojan L: Lymph vessel density in seminomatous testicular cancer assessed with the specific lymphatic endothelium cell markers D2-40 and LYVE-1: Correlation with pathologic parameters and clinical outcome. Urol Oncol 31: 1386-1394, 2013.

11. Feng Y, Wang W, Hu J, Ma J, Zhang Y and Zhang J: Expression of VEGF-C and VEGF-D as significant markers for assessment of lymphangiogenesis and lymph node metastasis in non-small cell lung cancer. Anat Rec 293: 802-812, 2010.

12. Tamura K, Matsushita M, Endo A, Kutsukake M and Kogo H: Effect of insulin-like growth factor-binding protein 7 on steroidogenesis in granulosa cells derived from equine chorionic gonadotropin-primed immature rat ovaries. Biol Reprod 77: 485-491, 2007

13. Ruan W, Xu E, Xu F, Ma Y, Deng H, Huang Q, Lv B, Hu H, Lin J, Cui J, et al: IGFBP7 plays a potential tumor suppressor role in colorectal carcinogenesis. Cancer Biol Ther 6: 354-359, 2007.

14. Wajapeyee N, Serra RW, Zhu X, Mahalingam M and Green MR: Oncogenic BRAF induces senescence and apoptosis through pathways mediated by the secreted protein IGFBP7. Cell 132: 363-374, 2008.

15. Pen A, Moreno MJ, Durocher $Y$, Deb-Rinker $P$ and Stanimirovic DB: Glioblastoma-secreted factors induce IGFBP7 and angiogenesis by modulating Smad-2-dependent TGF-beta signaling. Oncogene 27: 6834-6844, 2008.

16. Huang YJ, Niu J, Liu Z, Wang LE, Sturgis EM and Wei Q: The functional $I G F B P 7$ promoter $-418 \mathrm{G}>\mathrm{A}$ polymorphism and risk of head and neck cancer. Mutat Res 702: 32-39, 2010.

17. Xu LB, Liu C, Gao GQ, Yu XH, Zhang R and Wang J: Nerve growth factor-beta expression is associated with lymph node metastasis and nerve infiltration in human hilar cholangiocarcinoma. World J Surg 34: 1039-1045, 2010.

18. Wang J, Guo Y, Zhang BC, Chen ZT and Gao JF: Induction of apoptosis and inhibition of cell migration and tube-like formation by dihydroartemisinin in murine lymphatic endothelial cells. Pharmacology 80: 207-218, 2007.

19. Zhang B, Wang J, Gao J, Guo Y, Chen X, Wang B, Gao J, Rao Z and Chen Z: Alternatively activated RAW264.7 macrophages enhance tumor lymphangiogenesis in mouse lung adenocarcinoma. J Cell Biochem 107: 134-143, 2009.

20. Chen Z, Wang T, Luo H, Lai Y, Yang X, Li F, Lei Y, Su C, Zhang X, Lahn BT, et al: Expression of nestin in lymph node metastasis and lymphangiogenesis in non-small cell lung cancer patients. Hum Pathol 41: 737-744, 2010.

21. Saban MR, Towner R, Smith N, Abbott A, Neeman M, Davis CA, Simpson C, Maier J, Mémet S, Wu XR, et al: Lymphatic vessel density and function in experimental bladder cancer. BMC Cancer 7: 219, 2007.

22. Sleeman JP and Thiele W: Tumor metastasis and the lymphatic vasculature. Int J Cancer 125: 2747-2756, 2009.

23. Wang J, Li K, Wang B and Bi J: Lymphatic microvessel density as a prognostic factor in non-small cell lung carcinoma: A metaanalysis of the literature. Mol Biol Rep 39: 5331-5338, 2012

24. Achen MG and Stacker SA: Molecular control of lymphatic metastasis. Ann NY Acad Sci 1131: 225-234, 2008.

25. Bièche I, Lerebours F, Tozlu S, Espie M, Marty M and Lidereau R: Molecular profiling of inflammatory breast cancer: Identification of a poor-prognosis gene expression signature. Clin Cancer Res 10: 6789-6795, 2004.

26. Wang Y, Zhang D, Zheng W, Luo J, Bai Y and Lu Z: Multiple gene methylation of nonsmall cell lung cancers evaluated with 3-dimensional microarray. Cancer 112: 1325-1336, 2008. 
27. Ramsauer M and D'Amore PA: Contextual role for angiopoietins and TGFbeta1 in blood vessel stabilization. J Cell Sci 120: 1810-1817, 2007.

28. Shersher DD, Vercillo MS, Fhied C, Basu S, Rouhi O, Mahon B, Coon JS, Warren WH, Faber LP, Hong E, et al: Biomarkers of the insulin-like growth factor pathway predict progression and outcome in lung cancer. Ann Thorac Surg 92: 1805-1811, 2011.

29. Nancarrow DJ, Clouston AD, Smithers BM, Gotley DC, Drew PA, Watson DI, Tyagi S, Hayward NK and Whiteman DC; Australian Cancer Study; Study of Digestive Health: Whole genome expression array profiling highlights differences in mucosal defense genes in Barrett's esophagus and esophageal adenocarcinoma. PLoS One 6: e22513, 2011.

30. Jacob K, Quang-Khuong DA, Jones DT, Witt H, Lambert S, Albrecht S, Witt O, Vezina C, Shirinian M, Faury D, et al: Genetic aberrations leading to MAPK pathway activation mediate oncogene-induced senescence in sporadic pilocytic astrocytomas. Clin Cancer Res 17: 4650-4660, 2011.

31. Hu S, Chen R, Man X, Feng X, Cen J, Gu W, He H, Li J, Chai Y and Chen Z: Function and expression of insulin-like growth factor-binding protein 7 (IGFBP7) gene in childhood acute myeloid leukemia. Pediatr Hematol Oncol 28: 279-287, 2011.

32. Laranjeira AB, de Vasconcellos JF, Sodek L, Spago MC, Fornazim MC, Tone LG, Brandalise SR, Nowill AE and Yunes JA: IGFBP7 participates in the reciprocal interaction between acute lymphoblastic leukemia and BM stromal cells and in leukemia resistance to asparaginase. Leukemia 26: 1001-1011, 2012.

33. Heesch S, Bartram I, Neumann M, Reins J, Mossner M, Schlee C, Stroux A, Haferlach T, Goekbuget N, Hoelzer D, et al: Expression of $I G F B P 7$ in acute leukemia is regulated by DNA methylation. Cancer Sci 102: 253-259, 2011.
34. Tomanek B, Iqbal U, Blasiak B, Abulrob A, Albaghdadi $\mathrm{H}$, Matyas JR, Ponjevic D and Sutherland GR: Evaluation of brain tumor vessels specific contrast agents for glioblastoma imaging. Neuro Oncol 14: 53-63, 2012

35. Iqbal U, Albaghdadi H, Luo Y, Arbabi M, Desvaux C, Veres T, Stanimirovic D and Abulrob A: Molecular imaging of glioblastoma multiforme using anti-insulin-like growth factor-binding protein-7 single-domain antibodies. Br J Cancer 103: 1606-1616, 2010.

36. Abu-Safieh L, Abboud EB, Alkuraya H, Shamseldin H, Al-Enzi S, Al-Abdi L, Hashem M, Colak D, Jarallah A, Ahmad H, et al: Mutation of $I G F B P 7$ causes upregulation of BRAF/MEK/ERK pathway and familial retinal arterial macroaneurysms. Am J Hum Genet 89: 313-319, 2011.

37. Shersher DD, Vercillo MS, Fhied C, Basu S, Rouhi O, Mahon B, Coon JS, Warren WH, Faber LP, Hong E, et al: Biomarkers of the insulin-like growth factor pathway predict progression and outcome in lung cancer. Ann Thorac Surg 92: 1805-1811, 2011.

38. Georges RB, Adwan H, Hamdi H, Hielscher T, Linnemann U and Berger MR: The insulin-like growth factor binding proteins 3 and 7 are associated with colorectal cancer and liver metastasis. Cancer Biol Ther 12: 69-79, 2011.

39. Chen D, Yoo BK, Santhekadur PK, Gredler R, Bhutia SK, Das SK, Fuller C, Su ZZ, Fisher PB and Sarkar D: Insulin-like growth factor-binding protein-7 functions as a potential tumor suppressor in hepatocellular carcinoma. Clin Cancer Res 17: 6693-6701, 2011. 